\title{
Teoría de falla bajo carga estática
}

\section{Theories of failure under static load}

*Arturo Cruz-Avilés ${ }^{[a]}$, Martín Ortiz-Domínguez ${ }^{[\mathrm{b}]}$, Yolanda Suarez-López ${ }^{[\mathrm{c}]}$, Emilio A. RiveraLanderos $^{[\mathrm{d}]}$, Claudia B. Lechuga Canto ${ }^{[\mathrm{el}]}$, Yira Muños-Sánchez ${ }^{[\mathrm{f}]}$, Francisca Santana-Robles ${ }^{[\mathrm{g}]}$ Claudia García-Pérez $^{[\mathrm{h}]}$.

\section{Resumen}

La falla es la pérdida de función de un elemento tanto por deformación (fluencia) como por separación de sus partes (fractura). Los mecanismos de falla dependen de la estructura microscópica del material y de la forma de sus enlaces atómicos. Para predecir la falla de materiales bajo cargas estáticas (se considera carga estática a aquella que no varía su magnitud ni dirección en el tiempo) y poder hacer diseños de elementos de máquinas confiables se han desarrollado varias teorías para grupos de materiales, basándose en observaciones experimentales. En el presente escrito se determinó el factor de seguridad a partir del esfuerzo de cedencia $\left(S_{y}\right)$, el esfuerzo efectivo de Von Mises $\left(\sigma^{\prime}\right)$, el esfuerzo cortante máximo $\left(\tau_{m}\right)$ y la energía de distorsión $\left(U_{d}\right)$ empleados en el análisis y diseño de elementos de máquinas y estructurales.

Palabras clave: Esfuerzos principales, Círculos de Mohr, Esfuerzo de von Mises, Esfuerzo cortante máximo, Energía de distorsión 


\begin{abstract}
Failure is the loss of function of an element both by deformation (creep) and by separation of its parts (fracture). The mechanisms of failure depend on the microscopic structure of the material and the shape of its atomic bonds. To predict the failure of materials under static loads (static load is considered to be one that does not change its magnitude or direction in time) and to be able to make designs of reliable machine elements, several theories for groups of materials have been developed, based on experimental observations. In the present paper the safety factor was determined from the yield strength $\left(S_{y}\right)$, the effective force of Von Mises $\left(\sigma^{\prime}\right)$, the maximum shear stress $\left(\tau_{m}\right)$ and distortion energy $\left(U_{d}\right)$ used in the analysis and design of machine and structural elements.
\end{abstract}

Key words: Principal stresses, Mohr's circle, von Mises stress, Maximum shear stress, Distortion energy

\title{
Introducción
}

Actualmente se sabe mucho más acerca de varios tipos de fallas, debido en gran medida a la mejora de las pruebas y las técnicas de medición. Las fallas dependen del material en cuestión de su resistencia a la compresión, a la tensión y al cortante. También dependen del tipo de carga ya sea estática o dinámica, asimismo de la presencia o ausencia de grietas en el material. El círculo de Mohr para el estado de esfuerzos en una prueba de tensión que se aplica lentamente a la pieza cargada de tensión pura, que causa un esfuerzo de tensión normal. Sin embargo, el círculo de Mohr muestra que también se presenta un esfuerzo cortante, el cual es exactamente de la mitad de la mitad de la magnitud del esfuerzo normal. El círculo de Mohr en una prueba de torsión donde se aplica lentamente a la pieza una carga de torsión pura que causa un esfuerzo cortante. Sin embargo, el círculo de Mohr se presenta un esfuerzo normal, el cual es exactamente igual al esfuerzo cortante. 


\section{Criterio de la máxima energía de distorsión}

Una pieza falla si cede y se distorsiona lo suficiente como para no funcionar adecuadamente. Una pieza también falla cuando se fractura y se parten. Cualquiera de estas condiciones es una falla, pero los mecanismos que las causan llegan a ser muy diferentes. Sólo los materiales dúctiles pueden ceder de manera significativa antes de fracturarse. Los materiales frágiles se fracturan sin cambiar su forma drásticamente. Las curvas de esfuerzo-deformación de cada tipo de material reflejan tal diferencia, como se observa en la figura 1.
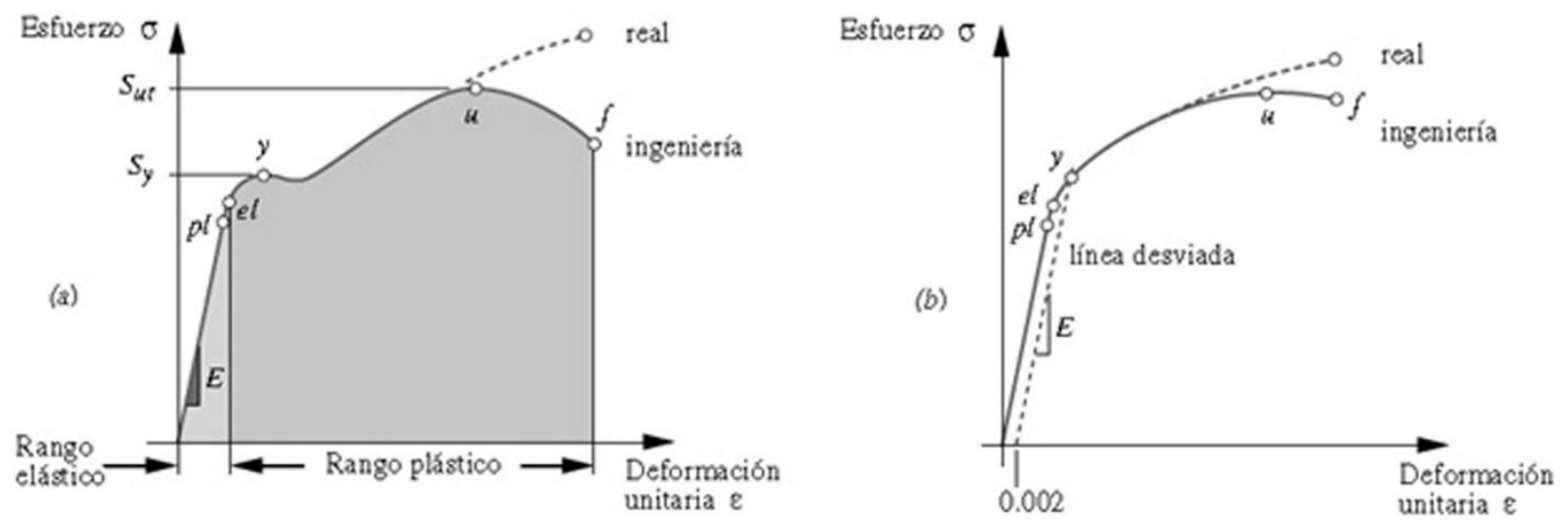

Figura 1. Curvas de ingeniería y de esfuerzo-deformación unitaria reales para materiales dúctiles: (a) Acero al bajo carbono; (b) Acero recocido al alto carbono.

(Norton, 2011)

La energía de deformación $U$ en unidades de volumen asociada con cualquier esfuerzo es el área bajo la curva de esfuerzo-deformación unitaria sea esencialmente hasta el punto de fluencia se representa con la siguiente ecuación.

$U=\frac{1}{2} \sigma \varepsilon$

Ampliando esto a un estado de esfuerzos tridimensional se obtiene lo siguiente:

$U=\frac{1}{2}\left(\sigma_{1} \varepsilon_{1}+\sigma_{2} \varepsilon_{2}+\sigma_{3} \varepsilon_{3}\right)$ 
Usando los esfuerzos principales y las deformaciones principales que actúan sobre los planos de esfuerzo cortante igual a cero. Esta expresión se plantea tan sólo en términos de esfuerzos principales, sustituyendo las relaciones se tiene:

$$
\begin{aligned}
& \varepsilon_{1}=\frac{1}{E}\left(\sigma_{1}-v \sigma_{2}-v \sigma_{3}\right) \\
& \varepsilon_{2}=\frac{1}{E}\left(\sigma_{2}-v \sigma_{1}-v \sigma_{3}\right) \\
& \varepsilon_{3}=\frac{1}{E}\left(\sigma_{3}-v \sigma_{2}-v \sigma_{1}\right)
\end{aligned}
$$

Sustituyendo las ecuaciones de la deformación unitaria en la energía total de deformación se obtiene:

$$
U=\frac{1}{2}\left(\frac{\sigma_{1}}{E}\left(\sigma_{1}-v \sigma_{2}-v \sigma_{3}\right)+\frac{\sigma_{2}}{E}\left(\sigma_{2}-v \sigma_{1}-v \sigma_{3}\right)+\frac{\sigma_{3}}{E}\left(\sigma_{3}-v \sigma_{2}-v \sigma_{1}\right)\right)
$$

Simplificando la Ec. (4), resulta en:

$$
U=\frac{1}{2 E}\left(\sigma_{1}\left(\sigma_{1}-v \sigma_{2}-v \sigma_{3}\right)+\sigma_{2}\left(\sigma_{2}-v \sigma_{1}-v \sigma_{3}\right)+\sigma_{3}\left(\sigma_{3}-v \sigma_{2}-v \sigma_{1}\right)\right)
$$

La Ec (5), se puede reescribir como:

$$
U=\frac{1}{2 E}\left(\sigma_{1}^{2}-v \sigma_{1} \sigma_{2}-v \sigma_{1} \sigma_{3}+\sigma_{2}^{2}-v \sigma_{1} \sigma_{2}-v \sigma_{2} \sigma_{3}+\sigma_{3}^{2}-v \sigma_{3} \sigma_{2}-v \sigma_{1} \sigma_{3}\right)
$$

Reduciendo términos de la Ec. (6) se tiene que;

$$
U=\frac{1}{2 E}\left(\sigma_{1}^{2}+\sigma_{2}^{2}+\sigma_{3}^{2}-2 \nu\left(\sigma_{1} \sigma_{2}+\sigma_{1} \sigma_{3}+\sigma_{2} \sigma_{3}\right)\right)
$$

donde $v$ es la razón de Poisson. 
Ahora sabemos que la energía total de deformación está compuesta por una energía de distorsión y una energía hidrostática como se representa en la siguiente ecuación;

$U=U_{h}+U_{d}$

También se puede expresar cada uno de los esfuerzos principales en términos de la componente hidrostática $\sigma_{h}$ que es común a cada cara, y una componente de distorsión $\sigma_{l d}$ que es única en cada cara, donde se representan las siguientes ecuaciones;

$$
\begin{aligned}
& \sigma_{1}=\sigma_{h}+\sigma_{d_{1}} \\
& \sigma_{2}=\sigma_{h}+\sigma_{d_{2}} \\
& \sigma_{3}=\sigma_{h}+\sigma_{d_{3}}
\end{aligned}
$$

Sumando los tres esfuerzos principales en la Ec. (9) se tiene;

$$
\begin{aligned}
& \sigma_{1}+\sigma_{2}+\sigma_{3}=3 \sigma_{h}+\sigma_{d_{1}}+\sigma_{d_{2}}+\sigma_{d_{3}} \\
& \sigma_{h}=\frac{1}{3}\left(\sigma_{1}+\sigma_{2}+\sigma_{3}-\left(\sigma_{d_{1}}+\sigma_{d_{2}}+\sigma_{d_{3}}\right)\right)
\end{aligned}
$$

Para un cambio volumétrico sin distorsión, el término negativo de la Ec. (10) es cero, dando así una expresión para la componente volumétrica o hidrostática $\sigma_{h}$.

$$
\sigma_{h}=\frac{\sigma_{1}+\sigma_{2}+\sigma_{3}}{3}
$$

En la cual notara que simplemente es un promedio de los tres esfuerzos principales en la Ec. (7) se escribe como: 


$$
\begin{aligned}
& U_{h}=\frac{1}{2 E}\left(\sigma_{h}^{2}+\sigma_{h}^{2}+\sigma_{h}^{2}-2 v\left(\sigma_{h}^{2}+\sigma_{h}^{2}+\sigma_{h}^{2}\right)\right) \\
& U_{h}=\frac{1}{2 E}\left(3 \sigma_{h}^{2}-2 v\left(3 \sigma_{h}^{2}\right)\right) \\
& U_{h}=\frac{3 \sigma_{h}^{2}}{2 E}(1-2 v)
\end{aligned}
$$

Sustituyendo la Ec. (11) en la Ec. (12) se tiene:

$$
U_{h}=\frac{3}{2 E}(1-2 v)\left(\frac{\sigma_{1}+\sigma_{2}+\sigma_{3}}{3}\right)^{2}
$$

Resolviendo la Ec. (13) se obtiene:

$$
U_{h}=\frac{3}{2 E}(1-2 v)\left(\frac{\left(\sigma_{1}+\sigma_{2}\right)^{2}+2\left(\sigma_{1}+\sigma_{2}\right) \sigma_{3+} \sigma_{3}^{2}}{9}\right)
$$

Reduciendo términos de la Ec. (14) se puede reescribir como:

$U_{h}=\frac{1}{6 E}(1-2 v)\left(\sigma_{1}^{2}+2\left(\sigma_{1} \sigma_{2}\right)+\sigma_{2}^{2}+2\left(\sigma_{1} \sigma_{2}\right) \sigma_{3}+\sigma_{3}^{2}\right)$

Simplificando la Ec. (15), se obtiene:

$U_{h}=\frac{(1-2 v)}{6 E}\left(\sigma_{1}^{2}+2\left(\sigma_{1} \sigma_{2}\right)+\sigma_{2}^{2}+2\left(\sigma_{1} \sigma_{2}\right) \sigma_{3}+\sigma_{3}^{2}\right)$

Se puede reescribir la Ec. (16) como:

$$
U_{h}=\frac{(1-2 v)}{6 E}\left(\sigma_{1}^{2}+\sigma_{2}^{2}+\sigma_{3}^{2}+2\left(\sigma_{1} \sigma_{2}+\sigma_{1} \sigma_{3}+\sigma_{2} \sigma_{3}\right)\right)
$$


Ahora para encontrar la energía de distorsión tenemos:

$U_{d}=U-U_{h}$

Sustituyendo la Ec. (6) y Ec. (17) en la Ec. (18) se tiene;

$$
\begin{aligned}
U_{d}=\left\{\left(\frac { 1 } { 2 E } \left(\sigma_{1}^{2}\right.\right.\right. & \left.\left.+\sigma_{2}^{2}+\sigma_{3}^{2}-2 v\left(\sigma_{1} \sigma_{2}+\sigma_{1} \sigma_{3}+\sigma_{2} \sigma_{3}\right)\right)\right) \\
& \left.-\left(\frac{(1-2 v)}{6 E}\left(\sigma_{1}^{2}+\sigma_{2}^{2}+\sigma_{3}^{2}+2\left(\sigma_{1} \sigma_{2}+\sigma_{1} \sigma_{3}+\sigma_{2} \sigma_{3}\right)\right)\right)\right\}
\end{aligned}
$$

Resolviendo la Ec. (19) se tiene que;

$$
\begin{aligned}
U_{d}=\left(\frac{\sigma_{1}^{2}+\sigma_{2}^{2}+\sigma_{3}^{2}}{2 E}-\frac{v\left(\sigma_{1} \sigma_{2}+\sigma_{1} \sigma_{3}+\sigma_{2} \sigma_{3}\right)}{E}\right) \\
-\left(\frac{\sigma_{1}^{2}+\sigma_{2}^{2}+\sigma_{3}^{2}}{6 E}+\frac{\left(\sigma_{1} \sigma_{2}+\sigma_{1} \sigma_{3}+\sigma_{2} \sigma_{3}\right)}{3 E}-\frac{v\left(\sigma_{1}^{2}+\sigma_{2}^{2}+\sigma_{3}^{2}\right)}{3 E}\right. \\
\left.\quad-\frac{2 v\left(\sigma_{1} \sigma_{2}+\sigma_{1} \sigma_{3}+\sigma_{2} \sigma_{3}\right)}{3 E}\right)
\end{aligned}
$$

Simplificando la Ec. (20) resulta en:

$$
\begin{aligned}
U_{d}=\left(\frac{\sigma_{1}^{2}+\sigma_{2}^{2}+\sigma_{3}^{2}}{2 E}-\frac{v\left(\sigma_{1} \sigma_{2}+\sigma_{1} \sigma_{3}+\sigma_{2} \sigma_{3}\right)}{E}\right) \\
+\left(-\frac{\sigma_{1}^{2}+\sigma_{2}^{2}+\sigma_{3}^{2}}{6 E}-\frac{\left(\sigma_{1} \sigma_{2}+\sigma_{1} \sigma_{3}+\sigma_{2} \sigma_{3}\right)}{3 E}+\frac{v\left(\sigma_{1}^{2}+\sigma_{2}^{2}+\sigma_{3}^{2}\right)}{3 E}\right. \\
\left.+\frac{2 v\left(\sigma_{1} \sigma_{2}+\sigma_{1} \sigma_{3}+\sigma_{2} \sigma_{3}\right)}{3 E}\right)
\end{aligned}
$$


Por otro lado la Ec. (21), se puede expresar como:

$$
\begin{gathered}
U_{d}=\frac{\sigma_{1}^{2}+\sigma_{2}^{2}+\sigma_{3}^{2}}{3 E}-\frac{v\left(\sigma_{1} \sigma_{2}+\sigma_{1} \sigma_{3}+\sigma_{2} \sigma_{3}\right)}{3 E}+\frac{v\left(\sigma_{1}^{2}+\sigma_{2}^{2}+\sigma_{3}^{2}\right)}{3 E} \\
-\frac{\left(\sigma_{1} \sigma_{2}+\sigma_{1} \sigma_{3}+\sigma_{2} \sigma_{3}\right)}{3 E}
\end{gathered}
$$

Reescribiendo la Ec. (22), se llega:

$U_{d}=\left(\frac{\nu+1}{3 E}\right)\left(\sigma_{1}^{2}+\sigma_{2}^{2}+\sigma_{3}^{2}\right)-\left(\frac{\nu+1}{3 E}\right)\left(\sigma_{1} \sigma_{2}+\sigma_{1} \sigma_{3}+\sigma_{2} \sigma_{3}\right)$

Simplificando la Ec. (23), se escribir como:

$U_{d}=\left(\frac{\nu+1}{3 E}\right)\left[\left(\sigma_{1}^{2}+\sigma_{2}^{2}+\sigma_{3}^{2}\right)-\left(\sigma_{1} \sigma_{2}+\sigma_{1} \sigma_{3}+\sigma_{2} \sigma_{3}\right)\right]$

El esfuerzo de falla de interés es la resistencia a la fluencia $S_{y}$. La energía de distorsión asociada a la fluencia en la prueba de tensión se calcula sustituyendo estos valores en la Ec. (24)

$U_{d}=\left(\frac{v+1}{3 E}\right) S_{y}^{2}$

Para el estado de esfuerzo bidimensional, $\sigma_{3}=0$ y $S_{y}$ se reduce a:

$S_{y}=\sqrt{\sigma_{1}^{2}+\sigma_{2}^{2}-\sigma_{1} \sigma_{2}}$

La ecuación bidimensional de la energía de distorsión Ec. (26) describe una elipse, la cual al graficarse sobre los ejes $\sigma_{1}, \sigma_{3}$ queda como se muestra en la Figura 2. El Interior de esta elipse define la región segura contra la fluencia bajo carga estática, para los esfuerzos biaxiales combinados. 


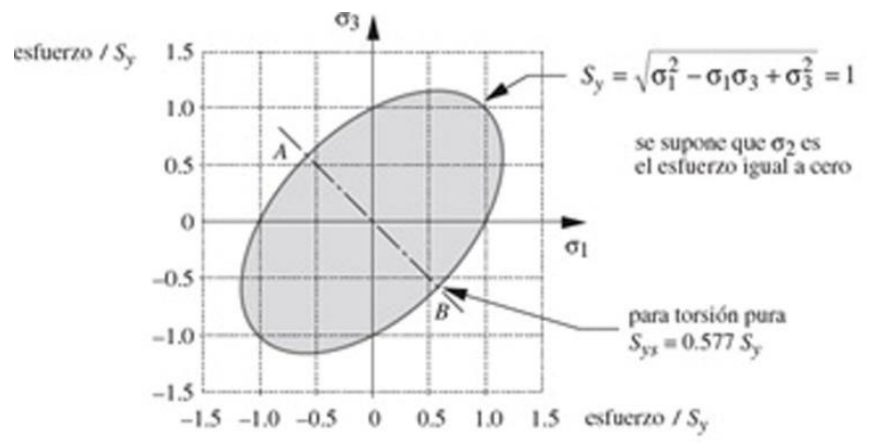

Figura 2. Elipse normalizada de la energía de distorsión bidimensional para la resistencia a la fluencia del material.

(Norton, 2011)

El esfuerzo efectivo de Von Mises $\boldsymbol{\sigma}$ ' se define como el esfuerzo de tensión uniaxial que crearía la misma energía de distorsión que la combinación real de los esfuerzos aplicados. Para el caso bidimensional partiendo de la Ec. (26) con $\sigma_{3}=0$, se expresa como:

$\sigma^{\prime}=\sqrt{\sigma_{1}^{2}+\sigma_{2}^{2}-\sigma_{1} \sigma_{2}}$

El esfuerzo de Von Mises en términos de los esfuerzos máximos y mínimos, se obtiene:

$\sigma_{1}^{2}=\left[\left(\frac{\sigma_{x}+\sigma_{y}}{2}\right)+\left(\left(\frac{\sigma_{x}-\sigma_{y}}{2}\right)^{2}+\tau_{x y}^{2}\right)^{\frac{1}{2}}\right]^{2}=\left(\sigma_{\text {prom }}+R\right)^{2}$

$\sigma_{2}^{2}=\left[\left(\frac{\sigma_{x}+\sigma_{y}}{2}\right)-\left(\left(\frac{\sigma_{x}-\sigma_{y}}{2}\right)^{2}+\tau_{x y}^{2}\right)^{\frac{1}{2}}\right]^{2}=\left(\sigma_{\text {prom }}-R\right)^{2}$ 
Multiplicando las Ec. (28) y Ec. (29) se tiene:

$\sigma_{1} \sigma_{2}=\left(\sigma_{\text {prom }}+R\right)\left(\sigma_{\text {prom }}-R\right)$

El esfuerzo de Von Mises $\sigma^{\prime}$ para el caso tridimensional es, a partir de la Ec. (27) se puede conocer lo siguiente:

$\sigma^{\prime}=\sqrt{\left(\sigma_{\text {prom }}+R\right)^{2}+\left(\sigma_{\text {prom }}-R\right)^{2}-\left(\sigma_{\text {prom }}+R\right)\left(\sigma_{\text {prom }}-R\right)}$

Resolviendo la Ec. (31) se obtiene:

$\sigma^{\prime}=$

$\sqrt{\begin{array}{r}\sigma_{\text {prom }}^{2}+2 \sigma_{\text {prom }} R+R^{2}+\sigma_{\text {prom }}^{2}-2 \sigma_{\text {prom }} R+R^{2} \\ -\left(\sigma_{\text {prom }}^{2}-\sigma_{\text {prom }} R+\sigma_{\text {prom }} R-R^{2}\right)\end{array}}$

Reduciendo términos de la Ec. (32) se puede reescribir como:

$\sigma^{\prime}=\sqrt{\sigma_{\text {prom }}^{2}+3 R^{2}}$

Debido a que $\sigma_{\text {prom }}^{2}=\left(\frac{\sigma_{x}+\sigma_{y}}{2}\right)^{2}$ y $R=\left(\left(\frac{\sigma_{x}-\sigma_{y}}{2}\right)^{2}+\tau_{x y}^{2}\right)^{\frac{1}{2}}$, sustituyendo en la Ec. (33) resulta:

$\sigma^{\prime}=\sqrt{\left(\frac{\sigma_{x}+\sigma_{y}}{2}\right)^{2}+3\left(\left(\left(\frac{\sigma_{x}-\sigma_{y}}{2}\right)^{2}+\tau_{x y}^{2}\right)^{\frac{1}{2}}\right)^{2}}$

Simplificando la Ec. (34), se obtiene: 
$\sigma^{\prime}=\sqrt{\left(\frac{\sigma_{x}+\sigma_{y}}{2}\right)^{2}+3\left(\frac{\sigma_{x}-\sigma_{y}}{2}\right)^{2}+3 \tau_{x y}^{2}}$

Resolviendo la Ec. (35) resulta en:

$\sigma^{\prime}=\sqrt{\frac{\sigma_{x}^{2}+2 \sigma_{x} \sigma_{y}+\sigma_{y}^{2}}{4}+\frac{3}{4}\left(\sigma_{x}^{2}+2 \sigma_{x} \sigma_{y}+\sigma_{y}^{2}\right)+3 \tau_{x y}^{2}}$

Reduciendo la Ec. (36) en términos de los esfuerzos aplicados, se tiene:

$\sigma^{\prime}=\sqrt{\sigma_{x}+\sigma_{y}-\sigma_{x} \sigma_{y}+3 \tau_{x y}^{2}}$

Teniendo en cuenta que:

$\frac{v+1}{3 E}=\frac{1}{6 G}$

Sustituyendo la Ec. (38) en la Ec. (25), se obtiene:

$U_{d}=\frac{S_{y}^{2}}{6 G}$

Con la ayuda de la Ec. (26), la Ec. (39) resulta en:

$U_{d}=\frac{1}{6 G}\left(\sigma_{1}^{2}+\sigma_{2}^{2}-\sigma_{1} \sigma_{2}\right)$

La energía de distorsión del cuerpo sebe ser menor a la energía del esfuerzo de cedencia:

$\left(\sigma_{1}^{2}+\sigma_{2}^{2}-\sigma_{1} \sigma_{2}<S_{y}^{2}\right)$ 
La energía de distorsión asociada con la fluencia en la prueba de tensión describe una elipse como lo muestra la Figura 3.

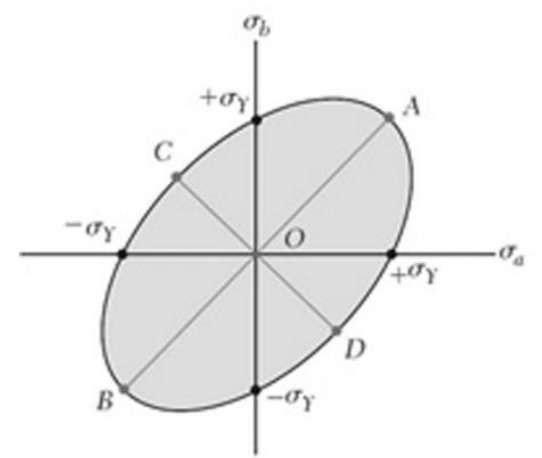

Figura 3. Área limitada por la elipse.

(Norton, 2011)

Esta área está limitada por la elipse cuya ecuación es:

$\left(\sigma_{1}^{2}+\sigma_{2}^{2}-\sigma_{1} \sigma_{2}=S_{y}^{2}\right)$

la cual interseca los ejes coordenados en $\sigma_{1}= \pm S_{y} y \sigma_{2}= \pm S_{y}$, Puede verificarse que el pie mayor de la elipse biseca el primer y tercer cuadrantes desde $\mathrm{A}\left(\sigma_{1}=\sigma_{2}=S_{y}\right)$, hasta $\mathrm{B} \sigma_{1}=\sigma_{2}=-S_{y}$, mientras su eje menor se extiende desde $\mathrm{C}\left(\sigma_{1}=-\sigma_{2}=-0.577 S_{y}\right)$ hasta $\mathrm{D}\left(\sigma_{1}=-\sigma_{2}=0.577 S_{y}\right)$ :

$\sigma_{1}^{2}+\sigma_{1}^{2}+\sigma_{1}^{2}=S_{y}^{2}$

Simplificando la Ec. (43), se obtiene:

$3 \sigma_{1}^{2}=S_{y}^{2}$

Aplicando raíz cuadrada en ambos lados y despejando $\sigma_{1}$ de la Ec. (44), se tiene:

$\sigma_{1}=\frac{S_{y}}{\sqrt{3}}=0.577 S_{y}$ 
Para efectos de diseño, resulta conveniente incluir un factor de seguridad F.S en los cálculos, de modo que el estado de esfuerzos sea seguro dentro de la elipse de falla:

$F . S=\frac{S_{y}}{\sigma^{\prime}}=\frac{S_{y}}{\sqrt{\sigma_{1}^{2}+\sigma_{2}^{2}-\sigma_{1} \sigma_{2}}}=\frac{S_{y}}{\sqrt{\sigma_{x}+\sigma_{y}-\sigma_{x} \sigma_{y}+3 \tau_{x y}^{2}}}$

Para aplicar el criterio del esfuerzo cortante máximo, en la Figura 4 se comparan el criterio del esfuerzo cortante máximo con el de la energía máxima de distorsión. Note que la elipse pasa por los vértices del hexágono. En consecuencia, para los estados de esfuerzo representados por estos seis puntos, los dos criterios coinciden. Para cualquier otro estado de esfuerzo, el criterio del esfuerzo cortante máximo es más conservador que el criterio de la máxima energía de distorsión, ya que el hexágono está dentro de la elipse. Establece que la falla ocurre cuando el esfuerzo cortante máximo en una pieza excede el esfuerzo cortante por fluencia en una muestra sujeta a tensión (la mitad de la resistencia de fluencia por tensión). Esto predice que la resistencia a la fluencia por cortante de un material es:

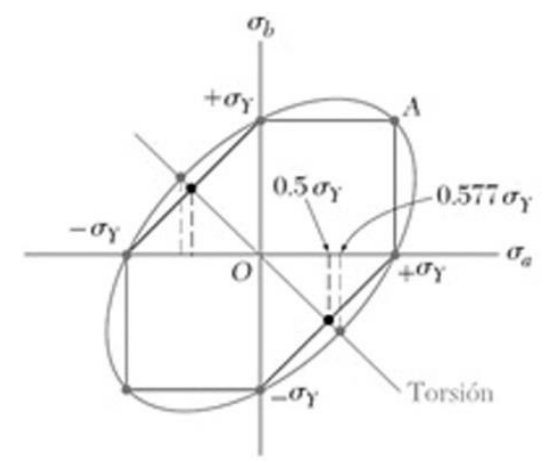

Figura 4. Comparación del criterio del esfuerzo cortante máximo con el de la energía máxima de distorsión. (Norton, 2011)

$S_{y s}=0.50 S_{y}$

Por lo tanto, el factor de seguridad es:

$F . S=\frac{S_{y s}}{\tau_{m}}=\frac{S_{y} / 2}{\left(\sigma_{1}^{2}-\sigma_{2}^{2}\right) / 2}=\frac{S_{y}}{\left(\sigma_{1}^{2}-\sigma_{2}^{2}\right)}$ 


\section{Resultados y discusiones}

Los materiales dúctiles e isotrópicos con cargas estáticas de tensión están limitadas por sus resistencias al cortante; mientras que los materiales frágiles están limitados por sus resistencias la tensión. Esta situación provoca que haya teorías de falla diferentes para las dos clases de materiales, dúctiles y frágiles.

La teoría del esfuerzo normal máximo establece que la falla ocurrirá cuando el esfuerzo normal en la muestra alcance algún límite de resistencia normal, tal como la resistencia a la fluencia por tensión a la resistencia última a la tensión. En materiales dúctiles, la resistencia a la fluencia es el criterio que se suele emplear.

\section{Conclusiones}

El objetivo de este trabajo fue determinar de manera detallada la teoría de falla bajo cargas estáticas empleadas en el análisis y diseño de elementos de máquinas y elementos estructurales. Asimismo, se determinó que la falla se presentará cuando el punto determinado por los esfuerzos se encuentra fuera del área sombreada de la Figura 7. La línea que delimita la elipse representa las locaciones donde se presentará la falla de acuerdo con von Mises, las líneas interiores representan las locaciones de falla de acuerdo con Tresca. Finalmente, en la misma Figura 7, se puede observar que la teoría de von Mises ( $\left.F . S=S_{y} / \sigma^{\prime}\right)$ tiene una mayor área en la cual no se presentará falla en comparación con la teoría de Tresca $\left(F . S=S_{y s} / \tau_{m}\right)$, por eso la teoría del esfuerzo cortante máximo es la teoría escogida para hacer los cálculos conservadores de falla de un material y tener una mayor certeza de que no se producirá falla.

\section{Agradecimientos}

El trabajo descrito en el presente artículo fue financiado por la beca otorgada por PRODEP. Asimismo, los autores le agradecen al Dr. Jorge Zuno Silva, quien es Director de la Escuela Superior de Ciudad Sahagún-UAEH, México, por todas las facilidades para concluir este trabajo de investigación. 


\section{Referencias}

Norton, R. L. (2011). Diseño de Máquinas. Estado de México, México: Prentice Hall Hispanoamérica, S. A.

Beer, F. P., Johnston, E. R., DeWolf, J. T. y Mazurek, D. F. (2013). Mecánica de Materiales. D. F., México: McGraw-Hill.

Pytel, A. y Singer, F. L. (1994). Resistencia de materiales. D. F., México: Oxford University Press México S.A. de C.V.

Shigley, J. E., and Mischke, C. R. (1989). Mechanical Engineering Design. New York, US: MacGraw-Hill.

${ }^{[a]}$ Licenciatura en Ingeniería Mecánica, Escuela Superior de Ciudad Sahagún, UAEH, Tepeapulco, Hidalgo, México, arturo_cruz8085@uaeh.edu.mx, 0000-0003-0455-1646;

${ }^{[b]}$ Licenciatura en Ingeniería Mecánica, Escuela Superior de Ciudad Sahagún, UAEH, Tepeapulco, Hidalgo, México, martin_ortiz@uaeh.edu.mx, 0000-0003-4475-9804;

${ }^{[c]}$ Licenciatura en Ingeniería Industrial, Escuela Superior de Ciudad Sahagún, UAEH, Tepeapulco, Hidalgo, México, yjuarez@uaeh.edu.mx;

${ }^{[\mathrm{d}]}$ Licenciatura en Contaduría, Escuela Superior de Ciudad Sahagún, UAEH, Tepeapulco, Hidalgo, México, rlandero@uaeh.edu.mx;

${ }^{[e]}$ Licenciatura en Contaduría, Escuela Superior de Ciudad Sahagún, UAEH, Tepeapulco, Hidalgo, México, claublc@uaeh.edu.mx;

${ }^{[f]}$ Licenciatura en Ingeniería Mecánica, Escuela Superior de Ciudad Sahagún, UAEH, Tepeapulco, Hidalgo, México, yira@uaeh.edu.mx;

${ }^{[\mathrm{g}]}$ Licenciatura en Ingeniería Industrial, Escuela Superior de Ciudad Sahagún, UAEH, Tepeapulco, Hidalgo, México, profe_7739@uaeh.edu.mx;

${ }^{[\mathrm{h}]}$ Licenciatura en Ingeniería Mecánica, Escuela Superior de Ciudad Sahagún, UAEH, Tepeapulco, Hidalgo, México, cgarciap@uaeh.edu.mx; 